\title{
Prevalence of Mild Cognitive Impairment in Rural Thai Older People, Associated Risk Factors and their Cognitive Characteristics
}

\author{
Jiranan Griffiths $^{\mathrm{a}} \quad$ Lakkana Thaikruea $^{\mathrm{a}} \quad$ Nahathai Wongpakaran $^{\mathrm{b}}$ \\ Peeraya Munkhetvit ${ }^{c}$ \\ aDepartment of Community Medicine, Faculty of Medicine, Chiang Mai University, \\ Chiang Mai, Thailand; 'b Department of Psychiatry, Faculty of Medicine, \\ Chiang Mai University, Chiang Mai, Thailand; ' ${ }^{\mathrm{D}}$ Department of Occupational Therapy, \\ Faculty of Associated Medical Sciences, Chiang Mai University, Chiang Mai, Thailand
}

\section{Keywords}

Aging and cognition - Mild cognitive impairment - Prevalence $\cdot$ Risk factors

\begin{abstract}
Introduction: Mild cognitive impairment $(\mathrm{MCl})$ is a transitional stage between normal cognition and dementia. A review showed that $10-15 \%$ of those with $\mathrm{MCl}$ annually progressed to Alzheimer's disease. Objective: This study aimed to investigate the prevalence and risk factors associated with $\mathrm{MCl}$ as well as the characteristics of cognitive deficits among older people in rural Thailand. Methods: A cross-sectional study in 482 people who were 60 years old and over was conducted in northern Thailand. The assessments were administered by trained occupational therapists using demographic and health characteristics, Mental Status Examination Thai 10, Activities of Daily Living - Thai Assessment Scale, 15-item Geriatric Depression Scale and the Montreal Cognitive Assessment-Basic (MoCA-B, Thai version). Results: The mean age of $\mathrm{MCl}$ was $68.3 \pm 6.82$ years, and most had an education $\leq 4$ years. The prevalence of $\mathrm{MCl}$ in older people was $71.4 \%$ (344 out of 482 ), and it increased with age. Low education and diabetes mellitus (DM) were the significant risk factors associated with cognitive decline. Older people with $\mathrm{MCl}$ were more likely to have an education $\leq 4$ years (RR $1.74,95 \% \mathrm{Cl} 1.21$ 2.51) and DM (RR 1.19, 95\% Cl 1.04-1.36) than those who did not. The 3 most common cognitive impairments according to MoCA-B were executive function (86\%), alternating attention (33.1\%) and delayed recall (31.1\%). Conclusion: The prevalence of $\mathrm{MCl}$ in older Thai people in a rural area is high compared with that in other countries. The explanation might be due to low education and underlying disease associated with $\mathrm{MCl}$. A suitable program that can reduce the prospects of $\mathrm{MCl}$ in rural Thailand is needed.




\section{Introduction}

A report from the World Alzheimer Organization in 2015 showed that the estimated number of individuals with dementia worldwide was 46.8 million. It was expected to rise to 131.5 million by 2050 and double every 20 years [1]. Mild cognitive impairment (MCI) is the transitional stage between normal cognition and dementia, and it is a prodromal condition which might be good predictive information for dementia such as Alzheimer disease (AD) [2]. Therefore, it is important to study the prevalence of MCI. Studies on the prevalence of MCI worldwide published in 2012 showed a range between 3 and $42 \%$ and a review in 2015 showed 5-36.7\% [3, 4]. Thailand is one of the countries in Southeast Asia where older people who were 60 years old and over reached $16 \%$ from a total population of 65.1 million in 2015 . With the proportion of those aged exceeding $10 \%$ of the total, Thailand has become an aging society [5, 6]. A few studies of the prevalence of MCI conducted in central Thailand reported ranges from 16.7 to $43.5 \%$, and the prevalence of cognitive impairment was high at $64.3 \%$ in north east Thailand. These numbers were different depending on the method of study which included tools, age, education and the areas of study [7-9]. It has been reported that higher age, heart problems, blood pressure and diabetes were considered as risk factors of MCI [10, 11]. Studies on the prevalence, risk factors and characteristics of cognitive impairment in rural northern Thailand are rare, and publications using the tool that has been validated for a very low educated population with Montreal Cognitive Assessment - Basic (MoCA-B, Thai version) have not yet been published worldwide. This study aimed to investigate the prevalence and risk factors associated with MCI including the characteristics of cognitive deficits among older people in rural Thailand. These results would be new information on identifying MCI in a rural area and could help early detection, intervention and monitoring in people who had a problem with MCI in a rural community-based setting.

\section{Materials and Methods}

A cross-sectional study was conducted with a total population of 1,096 older people among 11 villages in northern Thailand. The sample size estimate was based on 15\% from a previous study [12], and the calculated number was 579 people. Stratified random sampling was used.

The participants who were aged 60 years and older were recruited. The inclusion and exclusion were based on Albert et al. [13] MCI diagnostic criteria combined with a comprehensive interview for past and concurrent underlying physical and mental illnesses in order to exclude cognitive impairment due to known medical conditions and medications or substances.

Albert et al. [13] diagnostic criteria for MCI are concerned with a change in cognition, impairment in one or more cognitive domains, preservation of independence in functional abilities and no dementia. The inclusion criteria were independent in activities of daily living and the MoCA-B, Thai version scores that were 24 and lower. The exclusion criteria were dementia, depression, a history of other psychiatric problems, cerebrovascular accident, Parkinson's disease, brain injury and physical disability. People who had thyroid disease, who were on sleeping and stress medicines and also people who had eye and hearing problems were also excluded. In total, 482 older people met the criteria of the study.

The instruments were demographic and health characteristics, Mental Status Examination Thai 10 [14], activity of daily living (ADL), Thai Assessment Scale [15], 15-item Geriatric Depression Scale $[16,17]$ and MoCA-B, Thai version. MoCA-B, Thai version was developed by collaboration between the MoCA Clinic and Institute in Canada and Prince Mahidol Award 
Foundation and the Faculty of Medicine of Chulalongkorn University, Thailand. It is a standard test which already had been validated as a screening tool for detecting MCI. It had been designed and adjusted for older people with low education and varying literacy. One point was added to the total score for $<4$ years of education, and 2 points were added for an education of $<4$ years and illiterate, the maximum score was 30 . The sensitivity was $81 \%$, and the specificity was $86 \%$. Test-retest reliability was $0.91(p<0.001)$, and internal consistency was 0.82 [18]. The assessments were administered by trained occupational therapists.

After interviewing demographic and health characteristics, the Geriatric Depression Scale was done to exclude depression. The test for screening dementia, Mental Status Examination Thai 10 was used to ensure that the participants were not demented. The ADL Thai Assessment Scale was also used to assess their function. The MoCA-B, Thai version was then utilised to screen for MCI participants. Associated risk factors were defined as potential risk factors. These included demographic data (e.g., gender, age, education), memory problems and health conditions (history of hypertension, diabetes mellitus [DM], heart disease) [10, 11, $19,20]$.

The study was approved by the Ethics Committee of the Faculty of Medicine, Chiang Mai University. Written informed consent was obtained. The investigators explained details of the study to the participants. After agreement to participate and getting informed consent, the research team started the interviews. The study was conducted in villages in a communitybased setting.

\section{Statistical Analysis}

The data were analysed with SPSS software (version 26) and Epi info ${ }^{\mathrm{TM}}$ (version 7) [21]. Descriptive statistics; frequency, percentage, mean and SD were used to analyse demographic data. Univariate analysis included Pearson's chi-square, student $t$ test, and relative risk (95\% CI). A $p$ value $<0.05$ was considered as statistically significant.

\section{Results}

In total, 482 older people were included in the study. The prevalence of MCI was $71.4 \%$ $(n=344)$. The mean age in the MCI group was higher than in the non-MCI group $668.33 \pm 6.82$ years; $64.77 \pm 3.94$ years, respectively). The prevalence increased with age, $60-69$ years was $65.1 \%, 70-79$ years was $83.8 \%$ and over 80 years was $100 \%$ (Table 1 ).

Older people who were 70 years and older were more likely to have MCI than the younger group (60-69 years); RR 1.35, 95\% CI 1.22-1.50). Older people with MCI were more likely to have a low education ( $\leq 4$ years; RR 1.74, 95\% CI 1.21-2.51). Older people who had DM were more likely to have MCI than those who did not (RR 1.19, 95\% CI 1.04-1.36) (Table 2).

The 3 most common cognitive impairments were executive function (EF; 86\%), alternating attention (33.1\%) and delayed recall (31.1\%) (Fig. 1).

\section{Discussion/Conclusion}

The prevalence of MCI in older Thai people in a rural area in this study using MoCA-B Thai version was high. Age, education and DM were the significant risk factors associated with MCI. The explanation might be because most older people in our study had a very low education, mostly only 4 years or less as shown in Table 1 (95.1\%). In addition, most of them were farmers $(65.1 \%)$ and had a very low income ( $\leq 270 \mathrm{USD} /$ month). The prevalence of MCI in our study was different from another study that had been conducted in central Thailand using 
Griffiths et al.: $\mathrm{MCl}$ in Rural Thai Older People

Table 1. Demographic and health characteristics of older people with MCI and non-MCI

\begin{tabular}{|c|c|c|c|c|c|}
\hline \multirow[t]{2}{*}{ Characteristics (total 482) } & \multicolumn{2}{|c|}{$\operatorname{MCI}(n=344 ; 71.4 \%)$} & \multicolumn{2}{|c|}{ Non-MCI ( $n=138 ; 28.6 \%)$} & \multirow[t]{2}{*}{$p$ value } \\
\hline & frequency & percent & frequency & percent & \\
\hline \multicolumn{6}{|l|}{ Gender } \\
\hline Female & 192 & 55.8 & 66 & 47.8 & \multirow[t]{2}{*}{$0.112^{\dagger}$} \\
\hline Male & 152 & 44.2 & 72 & 52.2 & \\
\hline \multicolumn{6}{|l|}{ Age, years, range } \\
\hline $60-69$ & 228 & 66.3 & 122 & 88.4 & \multirow[t]{3}{*}{$0.001^{*, \dagger}$} \\
\hline $70-79$ & 83 & 24.1 & 16 & 11.6 & \\
\hline $80-89$ & 33 & 9.6 & - & - & \\
\hline Age, years, mean \pm SD & \multicolumn{2}{|c|}{$68.33 \pm 6.82$} & \multicolumn{2}{|c|}{$64.77 \pm 3.94$} & $0.001^{*, キ}$ \\
\hline \multicolumn{6}{|l|}{ Marital status } \\
\hline Single & 11 & 3.20 & 6 & 4.3 & \multirow[t]{3}{*}{$0.128^{\dagger}$} \\
\hline Married & 244 & 70.9 & 108 & 78.3 & \\
\hline Widow/divorced & 89 & 25.9 & 24 & 17.4 & \\
\hline \multicolumn{6}{|l|}{ Family type } \\
\hline Single family & 267 & 77.6 & 104 & 75.4 & \multirow[t]{2}{*}{$0.60^{\dagger}$} \\
\hline Extended family & 77 & 22.4 & 34 & 24.6 & \\
\hline \multicolumn{6}{|l|}{ Education, years } \\
\hline$\leq 4$ & 327 & 95.1 & 115 & 83.3 & \multirow[t]{2}{*}{$0.001^{*, \dagger}$} \\
\hline$>4$ & 17 & 4.9 & 23 & 16.7 & \\
\hline \multicolumn{6}{|l|}{ Occupation } \\
\hline Farmers & 224 & 65.1 & 81 & 58.7 & \multirow[t]{2}{*}{$0.006^{*, \dagger}$} \\
\hline Employees & 108 & 31.4 & 42 & 30.4 & \\
\hline \multicolumn{6}{|c|}{ Others; merchant, government } \\
\hline officer, not specified & 12 & 3.5 & 15 & 10.9 & \\
\hline \multicolumn{6}{|l|}{ Income, USD/month } \\
\hline$\leq 270$ & 327 & 95.1 & 122 & 88.4 & \multirow[t]{2}{*}{$0.009 *,+$} \\
\hline$>270$ & 17 & 4.9 & 16 & 11.6 & \\
\hline \multicolumn{6}{|l|}{ Hypertension } \\
\hline Yes & 172 & 50 & 68 & 49.3 & \multirow[t]{2}{*}{$0.886^{\dagger}$} \\
\hline No & 172 & 50 & 70 & 50.7 & \\
\hline \multicolumn{6}{|l|}{ DM } \\
\hline Yes & 49 & 14.2 & 10 & 7.2 & \multirow[t]{2}{*}{$0.034^{*+}$} \\
\hline No & 295 & 85.8 & 128 & 92.8 & \\
\hline \multicolumn{6}{|l|}{ Heart disease } \\
\hline Yes & 15 & 4.4 & 7 & 5.1 & $0.735^{\dagger}$ \\
\hline No & 329 & 95.6 & 131 & 94.9 & \\
\hline Memory problems & & & & & \\
\hline Yes & 200 & 58.1 & 71 & 51.4 & $0.181^{\dagger}$ \\
\hline No & 144 & 41.9 & 67 & 48.6 & \\
\hline $\begin{array}{l}\text { * Statistically significant } \\
\text { † Pearson's chi-square. } \\
\text { ‡ Student } t \text { test. } \\
\text { MCI, mild cognitive impa }\end{array}$ & Hetes inte & & & & \\
\hline
\end{tabular}

MoCA Thai version that showed lower prevalences (43.5\%) in a younger group that had a mean age of $\mathrm{MCI}$ at $57.12 \pm 7.48$ years and had higher income and a better education [8]. Compared to other countries, the results were consistent with the study in Saudi Arabia using MoCA Saudi Arabian version where the assessment was also done in a community-based setting. Their overall prevalence of cognitive impairment was $72.5 \%$, and the mean age of the sample group was $67 \pm 6$ years. However, after adjustment of the education-cutoff scores, their prevalence of MCI was reduced to $38.6 \%$. The Saudi Arabian study also showed that 
Table 2. Risk factors associated with MCI

\begin{tabular}{lllll}
\hline Factors & & RR & $95 \%$ CI & $p$ value \\
\hline Gender & Female/male & 1.10 & $0.98-1.23$ & 0.112 \\
Age & 70 up/60-69 years & 1.35 & $1.22-1.50$ & $0.001^{*}$ \\
Education & $\leq 4 />4$ years & 1.74 & $1.21-2.51$ & $0.001^{*}$ \\
Hypertension & Yes/no & 1.01 & $0.90-1.13$ & 0.886 \\
DM & Yes/no & 1.19 & $1.04-1.36$ & $0.034^{*}$ \\
Heart disease & Yes/no & 0.95 & $0.71-1.28$ & 0.735 \\
Memory problems & Yes/no & 1.08 & $0.96-1.22$ & 0.181 \\
\hline
\end{tabular}

* Statistically significant $(p<0.05)$.

MCI, mild cognitive impairment; DM, diabetes mellitus.

Fig. 1. Percentage of most common cognitive impairments found in MoCA-B in older people with MCI. MoCA-B, Montreal Cognitive Assessment-Basic; MCI, mild cognitive impairment.

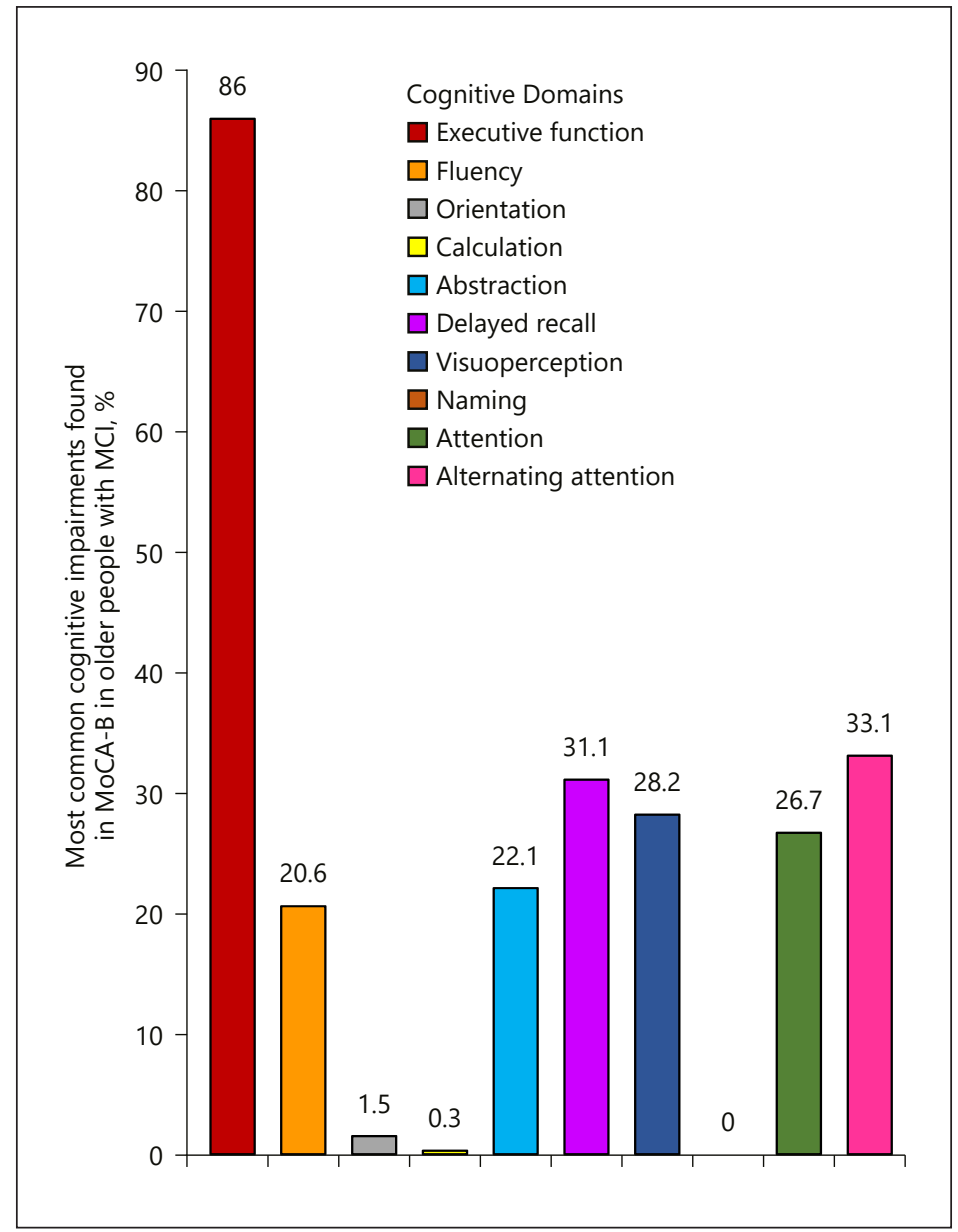

$57.6 \%$ of people with MCI had an education of $>7$ years [19]. However, the MoCA-B (Thai version) was already developed for screening MCI in illiterate and low education populations [18]. A study in China found that the prevalence of MCI was $20 \%$, and it was significantly higher in people who did not exercise [10]. The study worldwide of the prevalence of MCI in diverse geographical and ethnocultural regions which analyzed data from 11 international cohort studies which included USA, Europe, Asia and Australia found that the estimation of 
MCI prevalence was 5.0-36.7\%. The prevalence increased with age and not completing high school increased the likelihood of MCI significantly; however, sex was not a factor. These risk factors were consistent with our study [4]. Another systematic review in 2012 on the disparity of prevalence estimates in MCI across countries showed 3-42\%, the highest prevalence was found in a French study with $42 \%$ and a mean age $\geq 65$ years. The second highest prevalence was in a Finland study with $38.4 \%$ with mean age of 68.8 years. A low prevalence was found in a US study at $3.2 \%$ with mean age of 74.6 years. However, they reported that one reason for the differences in prevalence varied with the operational criteria of MCI and research methods. Petersen et al. [22] criteria were frequently cited in their review. It was also found that subjective memory complaints were not always included in the criteria, and no instruments were specified in the criteria [3]. In our study, 58.1\% accepted that they had memory problems.

In addition, DM and hypertension were risk factors associated with MCI, but only DM was a related significant risk factor. Heart disease was not a significant factor. The study in China demonstrated that stroke and DM but not hypertension and coronary heart disease were related risk factors of MCI [10]. Another Chinese study found that DM was shown to be a risk factor of $\mathrm{MCI}$ progressing to $\mathrm{AD}$. There was evidence suggesting that having type $2 \mathrm{DM}$ might be a potential risk in the progress from MCI to AD because DM is associated with factors such as obesity, hypertension, inflammation and dyslipidemia which were shown to have a negative impact on the brain [20]. Memory problems in older people in our study were not a significant risk factor associated with MCI. Likewise, the results in Saudi Arabia reported only $37.4 \%$ had memory complaints and were not shown as a risk factor in their results [19].

Considering the cognitive domains in our study assessed by MoCA-B Thai version, EF, alternating attention and delayed recall were most common cognitive impairments. This result was consistent with the concept of MCI by Petersen et al. [22] which revealed that the impairment of cognitive domains such as alteration in attention abilities, dysexecutive syndrome were found in MCI and an impairment of memory including delayed recall were found in early AD. Also other study indicated that decline in EF including attention may be indicative of several neurodegenerative conditions [23]. Another study also suggested that using working memory and EF could be used to assess or track the behavioural change to distinguish between normal aging, MCI and AD [24]. EF was associated with functional impairment or instrumental ADL in older people $[25,26]$.

The strength of our study was that we could include a large number of participants in the study in a rural northern Thailand community-based setting. However, the limitation was that there were a limited number of cognitive assessment tools that have been validated in a large sample size in Thailand to be used.

In conclusion, the results in this study provided early detection of MCI which needs to be done in community-based setting because the opportunity to access a specialist cognitive assessments in rural is likely to be less than a big town. This information of such early detection could be used to support health professionals to prepare a suitable program for $\mathrm{MCI}$. It is suggested that education is a significant risk factor associated with MCI. Conventional education may not be suitable for their age and experience. Therefore, the support should be an interaction with them using easy literacy activities that they can use in their daily life, outside a conventional classroom education that follows their interests and enjoyment. There is also a need to control DM, increase exercise and other physical activities in order to decrease related risk factors on MCI. In addition, older people should receive multifaceted cognitive training that may improve their cognition to reduce the prospect of MCI. 


\section{Acknowledgements}

The authors would like to gratefully acknowledge to the Faculty of Medicine, Chiang Mai University, Thailand, for funding this study and all the participants who contributed in this study.

\section{Statement of Ethics}

The research study has been approved by Ethics Committee from the Faculty of Medicine, Chiang Mai University, Thailand, which used international guidelines accordance with the World Medicine Association Declaration of Helsinki; study code COM-2561-05259. All the participants were given an overview of the research study and were informed that they could withdraw from the study if they did not feel comfortable. All of them were willing to participate in the study and have given their written informed consent.

\section{Disclosure Statement}

The authors declare that they have no competing financial interests.

\section{Funding Sources}

This study was supported by grant from the Faculty of Medicine, Chiang Mai University, Thailand (Grant number 108/2561).

\section{Author Contributions}

J.G.: first or main author performed the research including writing the proposal and the manuscript, designed research and planned the implementation of the research, collected data, prepared data, analysed data, wrote the results and discussed the results. L.T.: second author and corresponding author: main supervisor in the research proposal, supervised research design methods and helped with the data analysis; supported in applying for the funding and helped revising the manuscript. N.W.: third author: co-supervised the research proposal, discussed the design, supervised measurement utilisation, scoping MCI theory, data interpretation and helped revising manuscript. P.M.: fourth author: co-supervised the research proposal, discussed the research design, supervised in the preparation of the research staff in collecting data and helped revising the manuscript.

\section{References}

1 Alzheimer's Disease International. World Alzheimer Report 2015: The global Impact of dementia, an analysis of prevalence, incidence, cost and trends. [October 04, 2015]. Available from: http://www.alz.co.uk/research/ world-report-2015.

2 Tangalos EG, Petersen RC. Mild Cognitive Impairment in Geriatrics. Clin Geriatr Med. 2018 Nov;34(4):563-89.

3 Ward A, Arrighi HM, Michels S, Cedarbaum JM. Mild cognitive impairment: disparity of incidence and prevalence estimates. Alzheimers Dement. 2012 Jan;8(1):14-21.

4 Sachdev PS, Lipnicki DM, Kochan NA, Crawford JD, Thalamuthu A, Andrews G, et al. The Prevalence of Mild Cognitive Impairment in Diverse Geographical and Ethnocultural Regions: The COSMIC Collaboration. PLoS One. 2015 Nov;10(11):e0142388. 
5 Foundation of Thai gerontology research and development institute. Situation of the Thai elderly 2015. Bangkok, Thailand Amarin printing and publishing; 2017.

6 Foundation of Thai gerontology research and development institute. Situation research of the Thai elderly 2016. Salaya, Puttamonthon, Nakorn Pathom: Printery; 2017.

7 Deetong T, Puapornpong P, Pumipichet S, Benyakorn S, Kitporntheranunt M, Kongsomboon K. Prevalnece and risk factors of mild cognitive impairment in menopausal women at HRH Princess Maha Chakri Sirindhorn Medical Center. Thai J of Obestetrics and Gynaecology. 2013;21(3):110-6.

8 Kengsakul M, Chaikittisilpa S, Hemrungrojn S, Panyakhamlerd K, Jaisamrarn U, Taechakraichana N. The factors associated with mild cognitive impairment (MCI) in surgical menopause women. J Med Assoc Thai. 2015 Apr;98(4):327-33.

9 Sangsirilak A. The prevalence of cognitive impairment in elderly. Med J Srisaket Surin Buriram Hospitals. 2016;31(2):121-8.

10 Wang Y, Song M, Yu L, Wang L, An C, Xun S, et al. Mild cognitive impairment: vascular risk factors in community elderly in four cities of Hebei Province, China. PLoS One. 2015 May;10(5):e0124566.

11 Tervo S, Kivipelto M, Hänninen T, Vanhanen M, Hallikainen M, Mannermaa A, et al. Incidence and risk factors for mild cognitive impairment: a population-based three-year follow-up study of cognitively healthy elderly subjects. Dement Geriatr Cogn Disord. 2004;17(3):196-203.

12 Muangpaisan W, Intalapaporn S, Assantachai P. Digit span and verbal fluency tests in patients with mild cognitive impairment and normal subjects in Thai-community. J Med Assoc Thai. 2010 Feb; 93(2):224-30.

13 Albert MS, DeKosky ST, Dickson D, Dubois B, Feldman HH, Fox NC, et al. The diagnosis of mild cognitive impairment due to Alzheimer's disease: recommendations from the National Institute on Aging-Alzheimer's Association workgroups on diagnostic guidelines for Alzheimer's disease. Alzheimers Dement. 2011 May; $7(3): 270-9$.

14 Boongerd P, editor. Interesting topic about dementia. Dementia Association of Thailand, Newsletter. 2018. pp. $1-4$.

15 Tantiritsak T, editor. Institute of Neurology, Department of Medical Services, Thailand. Clinical Practice Guidelines: Dementia. Bangkok: Tana Press; 2014.

16 Sheikh JI, Yesavage JA. Geriatric Depression Scale; recent findings and development of a short version. In: Brink T, editor. Clin gerontol: a guide to assessment and intervention. 1986.

17 Wongpakaran N, Wongpakaran T. Prevalence of major depressive disorders and suicide in long-term care facilities: a report from northern Thailand. Psychogeriatrics. 2012 Mar;12(1):11-7.

18 Julayanont P, Tangwongchai S, Hemrungrojn S, Tunvirachaisakul C, Phanthumchinda K, Hongsawat J, et al. The Montreal Cognitive Assessment-Basic: A Screening Tool for Mild Cognitive Impairment in Illiterate and LowEducated Elderly Adults. J Am Geriatr Soc. 2015 Dec;63(12):2550-4.

19 Alkhunizan M, Alkhenizan A, Basudan L. Prevalence of Mild Cognitive Impairment and Dementia in Saudi Arabia: A Community-Based Study. Dement Geriatr Cogn Disord Extra. 2018 Mar;8(1):98-103.

20 Li W, Wang T, Xiao S. Type 2 diabetes mellitus might be a risk factor for mild cognitive impairment progressing to Alzheimer's disease. Neuropsychiatr Dis Treat. 2016 Sep;12:2489-95.

21 Epi infoTM: Centers for disease and control and prevention, Atlanta, GA; 2018. Available from: https://www. cdc.gov/epiinfo/user-guide/getting-started/acknowledgements.html.

22 Petersen RC, Doody R, Kurz A, Mohs RC, Morris JC, Rabins PV, et al. Current concepts in mild cognitive impairment. Arch Neurol. 2001 Dec;58(12):1985-92.

23 Reinvang I, Grambaite R, Espeseth T. Executive Dysfunction in MCI: Subtype or Early Symptom. Int J Alzheimers Dis. 2012;2012:936272.

24 Kirova AM, Bays RB, Lagalwar S. Working memory and executive function decline across normal aging, mild cognitive impairment, and Alzheimer's disease. BioMed Res Int. 2015;2015:748212.

25 Marshall GA, Rentz DM, Frey MT, Locascio JJ, Johnson KA, Sperling RA; Alzheimer's Disease Neuroimaging Initiative. Executive function and instrumental activities of daily living in mild cognitive impairment and Alzheimer's disease. Alzheimers Dement. 2011 May;7(3):300-8.

26 Brandt J, Aretouli E, Neijstrom E, Samek J, Manning K, Albert MS, et al. Selectivity of executive function deficits in mild cognitive impairment. Neuropsychology. 2009 Sep;23(5):607-18. 